\title{
$\underline{\mathbf{P}-111}$
}

\section{Flavonoid Analogues Isolated from the Stem Bark of Malaysian Polyalthia Cauliflora VAR. Cauliflora (Annonaceae) with Anticancer Properties}

\author{
Nurunajah Ab Ghani ${ }^{1,2, *}$, Norizan Ahmat ${ }^{1,2}$, Nor Hadiani Ismail ${ }^{1,2}$, Asmah Alias ${ }^{1}$, Nik Khairunissa \\ Nik Abdullah Zawawi ${ }^{1}$ and Siti Khadijah Sulaiman ${ }^{1}$ \\ ${ }^{I}$ Faculty of Applied Sciences, Universiti Teknologi MARA, 40450 Shah Alam, Malaysia; ${ }^{2}$ Atta-ur-Rahman Institute for \\ Natural Product Discovery, Level 9, FF3, Universiti Teknologi MARA, Puncak Alam Campus, 42300 Bandar Puncak \\ Alam, Selangor; E-mail: nurunajah@gmail.com
}

Phytochemical study on the stem bark of Polyalthia cauliflora var. cauliflora obtained from Pahang, Malaysia has been conducted. The separation of the chemical components were carried out by various chromatography techniques and their structures were elucidated by spectroscopic methods including nuclear magnetic resonance (1D and 2D NMR) as well as gas chromatography-mass spectrometry. Five flavones and two chalcones were isolated and has been identified as 3,7-dimethoxy5-hydroxyflavone (1), 5,8-dihydroxy-6,7-dimethoxyflavone (2), tetrochrysin (3), 6,7-dimethoxy-5-hydroxyflavone (4), 5hydroxy-3,7,8-trimethoxyflavone (5), 2',4'-dihydroxy-3'-methoxychalcone (6) and 2',6'-dihydroxychalcone (7). Isolated chalcone, 2',4'-dihydroxy-3'-methoxychalcone (6) was tested against three cell lines. Compound (6) shows active activity against HL-60 with $\mathrm{IC}_{50}=5.1 \mu \mathrm{g} / \mathrm{ml}$ and moderate activity with $\mathrm{IC}_{50} 12.2 \mu \mathrm{g} / \mathrm{ml}$ and $12.5 \mu \mathrm{g} / \mathrm{ml}$ against HeLa and MCF-7, respectively.

Keyword: Annonaceae, Polyalthia cauliflora, flavonoid, chalcone, NMR. 